\title{
Characterization, stability and hyperstability of multi-quadratic-cubic mappings
}

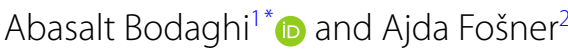

${ }^{\text {*Correspondence: }}$

abasalt.bodaghi@gmail.com

'Department of Mathematics, Garmsar Branch, Islamic Azad

University, Garmsar, Iran

Full list of author information is

available at the end of the article

\begin{abstract}
In this paper, we unify the system of functional equations defining a multi-quadratic-cubic mapping to a single equation. Applying a fixed point theorem, we study the generalized Hyers-Ulam stability of multi-quadratic-cubic mappings. As a result, we investigate the hyperstability of multi-quadratic-cubic mappings in some senses.
\end{abstract}

MSC: 39B52; 39B72; 39B82; 46B03

Keywords: Banach space; Hyers-Ulam stability; Multi-quadratic mapping; Multi-cubic mapping; Multi-quadratic-cubic mapping

\section{Introduction}

Let $V$ be a commutative group, $W$ be a linear space, and $n \geq 2$ be an integer. Recall from [14] that a mapping $f: V^{n} \longrightarrow W$ is called multi-additive if it is additive (satisfies Cauchy's functional equation $A(x+y)=A(x)+A(y)$ ) in each variable (see also [16]). Some facts on such mappings can be found in [24] and many other sources. Moreover, $f$ is said to be multi-quadratic if it is quadratic in each variable [15] (satisfies quadratic functional equation $Q(x+y)+Q(x-y)=2 Q(x)+2 Q(y)$ in each variable). In [32], Zhao et al. proved that the mapping $f: V^{n} \longrightarrow W$ is multi-quadratic if and only if the following relation holds:

$$
\sum_{s \in\{-1,1\}^{n}} f\left(x_{1}+s x_{2}\right)=2^{n} \sum_{j_{1}, j_{2}, \ldots, j_{n} \in\{1,2\}} f\left(x_{1 j_{1}}, x_{2 j_{2}}, \ldots, x_{n j_{n}}\right),
$$

where $x_{j}=\left(x_{1 j}, x_{2 j}, \ldots, x_{n j}\right) \in V^{n}$ with $j \in\{1,2\}$. Various versions of multi-quadratic mappings which were recently studied can be found in [9] and [30].

The first author and Shojaee introduced the multi-cubic mappings for the first time in [10]. In fact, a mapping $f: V^{n} \longrightarrow W$ is called multi-cubic if it is cubic in each variable, i.e., satisfies the equation

$$
C(2 x+y)+C(2 x-y)=2 C(x+y)+2 C(x-y)+12 C(x)
$$

in each variable. Indeed, the cubic functional equation (1.2) was introduced and studied by Jun and Kim in [22]. In [10], the authors unified the system of functional equations defining a multi-cubic mapping to a single equation, namely, a multi-cubic functional equation.

(c) The Author(s) 2021. This article is licensed under a Creative Commons Attribution 4.0 International License, which permits use sharing, adaptation, distribution and reproduction in any medium or format, as long as you give appropriate credit to the original author(s) and the source, provide a link to the Creative Commons licence, and indicate if changes were made. The images or other third party material in this article are included in the article's Creative Commons licence, unless indicated otherwise in a credit line to the material. If material is not included in the article's Creative Commons licence and your intended use is not permitted by statutory regulation or exceeds the permitted use, you will need to obtain permission directly from the copyright holder. To view a copy of this licence, visit http://creativecommons.org/licenses/by/4.0/. 
The motivation for many researchers to study the stability of functional equations is due to the query framed by Ulam [31] in 1940 as the stability of group homomorphisms: "when is it true that the solution of an equation differing slightly from a given one, must of necessity be close to the solution of the given equation?" The first partial answer, in the case of Cauchy's equation in Banach spaces, to Ulam's question was given by Hyers [21] stability involving a positive constant). Later, the result of Hyers was significantly generalized by Aoki [1], Th. M. Rassias [29] (stability incorporated with sum of powers of norms), Găvruța [20] (stability controlled by a general control function) and [27] (stability including mixed product-sum of powers of norms). A functional equation $\Gamma$ is said to be hyperstable if any function $f$ satisfying the equation $\Gamma$ approximately (in some senses) must be actually solutions to it. It seems that the first hyperstability result was published in [11] and concerned the ring homomorphisms. However, The term hyperstability has been used for the first time in [25]. In [14] and [15], Ciepliński studied the generalized Hyers-Ulam stability of multi-additive and multi-quadratic mappings in Banach spaces, respectively (see also [17] and [32]). In [10], it is shown that every multi-cubic functional equation is stable and moreover such functional equations under some conditions can be hyperstable (for the miscellaneous versions of multi-cubic mappings and their stabilities, we refer to [19] and [26]). Other forms of cubic functional equations and their stabilities can be found in $[4-7,18,23,28]$.

Motivated by [10] and [32], in this paper, we define multi-quadratic-cubic mappings and present a characterization of such mappings. In other words, we reduce the system of $n$ equations defining the multi-quadratic-cubic mappings to obtain a single functional equation. We also prove the generalized Hyers-Ulam stability and hyperstability for multiquadratic-cubic functional equations by using the fixed point method which was used for the first time by Brzdęk in [12]; for more applications of this approach for the stability of multi-Cauchy-Jensen, multi-additive-quadratic, multi-cubic and multi-quartic mappings in Banach spaces see $[2,3,10]$ and [8] respectively.

\section{Characterization of multi-quadratic-cubic mappings}

Throughout this paper, $\mathbb{N}, \mathbb{Z}$ and $\mathbb{Q}$ are the set of all positive integers, integers and rational numbers, respectively, $\mathbb{N}_{0}:=\mathbb{N} \cup\{0\}, \mathbb{R}_{+}:=[0, \infty)$. For any $l \in \mathbb{N}_{0}, n \in \mathbb{N}, t=\left(t_{1}, \ldots, t_{n}\right) \in$ $\{-1,1\}^{n}$ and $x=\left(x_{1}, \ldots, x_{n}\right) \in V^{n}$ we write $l x:=\left(l x_{1}, \ldots, l x_{n}\right)$ and $t x:=\left(t_{1} x_{1}, \ldots, t_{n} x_{n}\right)$, where $l x$ stands, as usual, for the scalar product of $l$ on $x$ in the linear space $V$.

Let $V$ and $W$ be linear spaces, $n \in \mathbb{N}$ and $k \in\{0, \ldots, n\}$. A mapping $f: V^{n} \longrightarrow W$ is called $k$-quadratic and $n-k$-cubic (briefly, multi-quadratic-cubic) if $f$ is quadratic in each of some $k$ variables and is cubic in each of the other variables (see Eq. (1.2)). In this note, we suppose for simplicity that $f$ is quadratic in each of the first $k$ variables, but one can obtain analogous results without this assumption. Let us note that, for $k=n(k=0)$, the above definition leads to the so-called multi-quadratic (multi-cubic) mappings.

In this section, we assume that $V$ and $W$ are vector spaces over $\mathbb{Q}$. Moreover, we identify $x=\left(x_{1}, \ldots, x_{n}\right) \in V^{n}$ with $\left(x^{k}, x^{n-k}\right) \in V^{k} \times V^{n-k}$, where $x^{k}:=\left(x_{1}, \ldots, x_{k}\right)$ and $x^{n-k}:=\left(x_{k+1}, \ldots, x_{n}\right)$, and we adopt the convention that $\left(x^{n}, x^{0}\right):=x^{n}:=\left(x^{0}, x^{n}\right)$. Put $x_{i}^{k}=$ $\left(x_{i 1}, \ldots, x_{i k}\right) \in V^{k}$ and $x_{i}^{n-k}=\left(x_{i, k+1} \cdots, x_{i n}\right) \in V^{n-k}$ where $i \in\{1,2\}$. We shall denote $x_{i}^{n}$ by $x_{i}$ if there is no risk of ambiguity. In addition, we put $\mathcal{M}=\left\{\mathfrak{N}_{n}=\left(N_{k+1}, \ldots, N_{n}\right) \mid N_{j} \in\right.$ $\left.\left\{x_{1 j} \pm x_{2 j}, x_{1 j}\right\}\right\}$, where $j \in\{k+1, \ldots, n\}$. Consider

$$
\mathcal{M}_{T}^{n-k}:=\left\{\mathfrak{N}_{n}=\left(N_{k+1}, \ldots, N_{n}\right) \in \mathcal{M} \mid \operatorname{Card}\left\{N_{j}: N_{j}=x_{1 j}\right\}=T\right\} .
$$


From now on, we use the following notations:

$$
\begin{aligned}
& f\left(\mathcal{M}_{T}^{n-k}\right):=\sum_{\mathfrak{N}_{n} \in \mathcal{M}_{T}^{n-k}} f\left(\mathfrak{N}_{n}\right), \\
& f\left(x_{i}^{k}, \mathcal{M}_{T}^{n-k}\right):=\sum_{\mathfrak{N}_{n} \in \mathcal{M}_{T}^{n-k}} f\left(x_{i}^{k}, \mathfrak{N}_{n}\right) \quad(i \in\{1,2\}) .
\end{aligned}
$$

In the next result, we reduce the system of $n$ equations defining the $k$-quadratic and $n-k$-cubic mapping to obtain a single functional equation.

Proposition 2.1 Let $n \in \mathbb{N}$ and $k \in\{0, \ldots, n\}$. If the mapping $f: V^{n} \longrightarrow W$ is $k$-quadratic and $n-k$-cubic mapping, then $f$ satisfies the equation

$$
\begin{gathered}
\sum_{s \in\{-1,1\}^{k}} \sum_{t \in\{-1,1\}^{n-k}} f\left(x_{1}^{k}+s x_{2}^{k}, 2 x_{1}^{n-k}+t x_{2}^{n-k}\right) \\
=2^{k} \sum_{m=0}^{n-k} 2^{n-k-m} 12^{m} \sum_{i \in\{1,2\}} f\left(x_{i}^{k}, \mathcal{M}_{m}^{n-k}\right)
\end{gathered}
$$

for all $x_{i}^{k}=\left(x_{i 1}, \ldots, x_{i k}\right) \in V^{k}$ and $x_{i}^{n-k}=\left(x_{i, k+1}, \ldots, x_{i n}\right) \in V^{n-k}$ where $i \in\{1,2\}$.

Proof Since for $k \in\{0, n\}$ our assertion follows from [10, Proposition 2.2] and [32, Theorem 3], we can assume that $k \in\{1, \ldots, n-1\}$. For any $x^{n-k} \in V^{n-k}$, define the mapping $g_{x^{n-k}}: V^{k} \longrightarrow W$ by $g_{x^{n-k}}\left(x^{k}\right):=f\left(x^{k}, x^{n-k}\right)$ for $x^{k} \in V^{k}$. By assumption, $g_{x^{n-k}}$ is $k$-quadratic, and hence Theorem 3 from [32] implies that

$$
\sum_{s \in\{-1,1\}^{k}} g_{x^{n-k}}\left(x_{1}^{k}+s x_{2}^{k}\right)=2^{k} \sum_{j_{1}, j_{2}, \ldots, j_{k} \in\{1,2\}} g_{x^{n-k}}\left(x_{j_{1} 1}, x_{j_{2} 2}, \ldots, x_{j_{k} k}\right), \quad\left(x_{1}^{k}, x_{2}^{k} \in V^{k}\right) .
$$

It now follows from the above equality that

$$
\sum_{s \in\{-1,1\}^{k}} f\left(x_{1}^{k}+s x_{2}^{k}, x^{n-k}\right)=2^{k} \sum_{j_{1}, j_{2}, \ldots, j_{k} \in\{1,2\}} f\left(x_{j_{1} 1}, x_{j_{2} 2}, \ldots, x_{j_{k} k}, x^{n-k}\right)
$$

for all $x_{1}^{k}, x_{2}^{k} \in V^{k}$ and $x^{n-k} \in V^{n-k}$. Similar to the above, for any $x^{k} \in V^{k}$, consider the mapping $h_{x^{k}}: V^{n-k} \longrightarrow W$ defined through $h_{x^{k}}\left(x^{n-k}\right):=f\left(x^{k}, x^{n-k}\right), x^{n-k} \in V^{n-k}$ which is $n-k$-cubic and so we conclude from Proposition 2.2 of [10] that

$$
\sum_{t \in\{-1,1\}^{n-k}} h_{x^{k}}\left(2 x_{1}^{n-k}+t x_{2}^{n-k}\right)=\sum_{m=0}^{n-k} 2^{n-k-m} 12^{m} h_{x^{k}}\left(\mathcal{M}_{k}^{n-k}\right)
$$

for all $x_{1}^{n-k}, x_{2}^{n-k} \in V^{n-k}$. By the definition of $h_{x^{k}}$, Eq. (2.3) is equivalent to

$$
\sum_{t \in\{-1,1\}^{n-k}} f\left(x^{k}, 2 x_{1}^{n-k}+t x_{2}^{n-k}\right)=\sum_{m=0}^{n-k} 2^{n-k-m} 12^{m} f\left(x^{k}, \mathcal{M}_{k}^{n-k}\right)
$$


for all $x_{1}^{n-k}, x_{2}^{n-k} \in V^{n-k}$ and $x^{k} \in V^{k}$. Plugging equality (2.2) into (2.4), we get

$$
\begin{aligned}
& \sum_{s \in\{-1,1\}^{k}} \sum_{t \in\{-1,1\}^{n-k}} f\left(x_{1}^{k}+s x_{2}^{k}, 2 x_{1}^{n-k}+t x_{2}^{n-k}\right) \\
& =\sum_{s \in\{-1,1\}^{k}} \sum_{m=0}^{n-k} 2^{n-k-m} 12^{m} f\left(x_{1}^{k}+s x_{2}^{k}, \mathcal{M}_{m}^{n-k}\right) \\
& =2^{k} \sum_{m=0}^{n-k} 2^{n-k-m} 12^{m} \sum_{j_{1}, j_{2}, \ldots, j_{k} \in\{1,2\}} f\left(x_{j_{1} 1}, x_{j_{2} 2}, \ldots, x_{j_{k} k}, \mathcal{M}_{m}^{n-k}\right) \\
& =2^{k} \sum_{m=0}^{n-k} 2^{n-k-m} 12^{m} \sum_{i \in\{1,2\}} f\left(x_{i}^{k}, \mathcal{M}_{m}^{n-k}\right)
\end{aligned}
$$

for all $x_{i}^{k}=\left(x_{i 1}, \ldots, x_{i k}\right) \in V^{k}$ and $x_{i}^{n-k}=\left(x_{i k+1}, \ldots, x_{i n}\right) \in V^{n-k}$, which proves that $f$ satisfies Eq. (2.1).

By a mathematical computation, one can check that the mapping $f: \mathbb{R}^{n} \longrightarrow \mathbb{R}$ defined through $f\left(z_{1}, \ldots, z_{n}\right)=\prod_{j=1}^{k} \prod_{i=k+1}^{n} z_{j}^{2} z_{i}^{3}$ satisfies (2.1) and so this equation is said to be multi-quadratic-cubic functional equation.

In the sequel, $\left(\begin{array}{l}n \\ k\end{array}\right)$ is the binomial coefficient defined for all $n, k \in \mathbb{N}_{0}$ with $n \geq k$ by $n ! /(k !(n-k) !)$.

Let $r \in \mathbb{N}$. We say the mapping $f: V^{n} \longrightarrow W$ satisfies the $r$-power condition in the $j$ th variable if

$$
f\left(z_{1}, \ldots, z_{j-1}, 2 z_{j}, z_{j+1}, \ldots, z_{n}\right)=2^{r} f\left(z_{1}, \ldots, z_{j-1}, z_{j}, z_{j+1}, \ldots, z_{n}\right), \quad\left(\left(z_{1}, \ldots, z_{n}\right) \in V^{n}\right) .
$$

Remark 2.2 It is easily verified that, if $f$ is a multi-cubic mapping, then it satisfies 3-power condition in all variables. But the converse is not true. Here, by means of an example we show that 3-power condition in all variables for a mapping $f$ does not imply that it is multicubic. Let $(\mathcal{A},\|\cdot\|)$ be a Banach algebra. Fix the vector $a_{0}$ in $\mathcal{A}$ (not necessarily unit). Define the mapping $h: \mathcal{A}^{n} \longrightarrow \mathcal{A}$ by $h\left(a_{1}, \ldots, a_{n}\right)=\prod_{j=1}^{n}\left\|a_{j}\right\|^{3} a_{0}$ for $\left(a_{1}, \ldots, a_{n}\right) \in \mathcal{A}^{n}$. It is easy to check that the mapping $h$ satisfies 3-power condition in all variables but $h$ is not multicubic even for $n=1$, that is, $h$ does not satisfy Eq. (1.2).

Let $0 \leq p \leq k$ and $0 \leq q \leq n-k$. Put

$$
\begin{aligned}
& \mathcal{K}_{(p, q)} \\
& \quad=\left\{(p, q) x:=(\overbrace{0, \ldots, 0, x_{i_{1}}, 0, \ldots, 0, x_{i_{p}}, 0, \ldots, 0}^{k \text {-times }}, \overbrace{0, \ldots, 0, x_{j_{1}}, 0, \ldots, 0, x_{j_{q}}, 0, \ldots, 0}^{n-k \text {-times }}) \in V^{n}\right\},
\end{aligned}
$$

where $1 \leq i_{1}<\cdots<i_{p} \leq k$ and $1 \leq j_{1}<\cdots<j_{q} \leq n-k$.

We wish to show that, if the mapping $f: V^{n} \rightarrow W$ satisfies Eq. (2.1), then it is multiquadratic-cubic. In order to do this, we present the next lemma.

Lemma 2.3 If the mapping $f: V^{n} \longrightarrow W$ satisfies Eq. (2.1) and the 3-power condition in the last $n-k$ variables, then $f(x)=0$ for any $x \in V^{n}$ with at least one component which is equal to zero. 
Proof We argue by induction on $p+q$ that, for each ${ }_{(p, q)} x \in \mathcal{K}_{(p, q)}, f\left({ }_{(p, q)} x\right)=0$ for $0 \leq p \leq k$ and $0 \leq q \leq n-k$. For $p+q=0$, by putting $\left(x_{1}^{k}, x_{1}^{n-k}\right)=\left(x_{2}^{k}, x_{2}^{n-k}\right)=(0, \ldots, 0)$ in (2.1), we have

$$
\begin{aligned}
2^{n} f(0, \ldots, 0) & =2^{k} \sum_{m=0}^{n-k}\left(\begin{array}{c}
n-k \\
m
\end{array}\right) 2^{n-k-m} 12^{m} 2^{k} 2^{n-k-m} f(0, \ldots, 0) \\
& =2^{2 n} \sum_{m=0}^{n-k}\left(\begin{array}{c}
n-k \\
m
\end{array}\right) 3^{m} f(0, \ldots, 0) \\
& =2^{2 n}(3+1)^{n-k} f(0, \ldots, 0) \\
& =2^{4 n-2 k} f(0, \ldots, 0) .
\end{aligned}
$$

It follows from (2.5) that $f(0, \ldots, 0)=0$. Assume that, for each ${ }_{(p, q)} x \in \mathcal{K}_{(p, q)}, f\left(_{(p, q)} x\right)=0$ with $p+q=s-1$. We show that, if ${ }_{(p, q)} x \in \mathcal{K}_{(p, q)}$, then $f\left({ }_{(p, q)} x\right)=0$ for $p+q=s$. By a suitable replacement in (2.1) and using the assumption, we get

$$
\begin{aligned}
2^{n} 2^{3 q} f\left({ }_{(p, q)} x\right) & =2^{k} \sum_{m=0}^{n-k-q}\left(\begin{array}{c}
n-k-q \\
m
\end{array}\right) 2^{n-k-m} 12^{m} 2^{k-p} 2^{n-k-m} f\left({ }_{(p, q)} x\right) \\
& =2^{2 n-p} \sum_{m=0}^{n-k-q}\left(\begin{array}{c}
n-k-q \\
m
\end{array}\right) 3^{m} f\left({ }_{(p, q)} x\right) \\
& \left.=2^{2 n-p}(3+1)^{n-k-q} f\left({ }_{(p, q)} x\right)=2^{4 n-2 k-p-2 q} f_{(p, q)} x\right) .
\end{aligned}
$$

Hence, $f\left({ }_{(p, q)} x\right)=0$. This shows that $f(x)=0$ for any $x \in V^{n}$ with at least one component which is equal to zero.

In the following result, we show that, if a mapping $f$ satisfies the functional equation (2.1), then it can be multi-quadratic-cubic under some mild conditions.

Proposition 2.4 If the mapping $f: V^{n} \longrightarrow W$ satisfies Eq. (2.1) and the 3-power condition in the last $n-k$ variables, then it is multi-quadratic-cubic.

Proof Putting $x_{2}^{n-k}=(0, \ldots, 0)$ in the left side of (2.1) and applying the hypothesis, we obtain

$$
2^{n-k} \times 2^{3(n-k)} \sum_{s \in\{-1,1\}^{k}} f\left(x_{1}^{k}+s x_{2}^{k}, x_{1}^{n-k}\right)=2^{4(n-k)} \sum_{s \in\{-1,1\}^{k}} f\left(x_{1}^{k}+s x_{2}^{k}, x_{1}^{n-k}\right)
$$

for all $x_{1}^{k}, x_{2}^{k} \in V^{k}$ and $x_{1}^{n-k} \in V^{n-k}$. On the other hand, the right side of (2.1) will be

$$
\begin{aligned}
2^{k} & \sum_{m=0}^{n-k}\left(\begin{array}{c}
n-k \\
m
\end{array}\right) 2^{n-k-m} 12^{m} 2^{n-k-m} \sum_{j_{1}, j_{2}, \ldots, j_{k} \in\{1,2\}} f\left(x_{j_{1}}, x_{j_{2} 2}, \ldots, x_{j_{k} k}, x_{1}^{n-k}\right) \\
& =2^{k} \sum_{m=0}^{n-k}\left(\begin{array}{c}
n-k \\
m
\end{array}\right) 4^{n-k-m} 12^{m} \sum_{j_{1}, j_{2}, \ldots, j_{k} \in\{1,2\}} f\left(x_{j_{1} 1}, x_{j_{2} 2}, \ldots, x_{j_{k} k}, x_{1}^{n-k}\right) \\
& =2^{4 n-3 k} \sum_{j_{1}, j_{2}, \ldots, j_{k} \in\{1,2\}} f\left(x_{j_{1} 1}, x_{j_{2} 2}, \ldots, x_{j_{k} k}, x_{1}^{n-k}\right)
\end{aligned}
$$


for all $x_{1}^{k}, x_{2}^{k} \in V^{k}$ and $x_{1}^{n-k} \in V^{n-k}$. Comparing Eqs. (2.6) and (2.7), we find

$$
\sum_{s \in\{-1,1\}^{k}} f\left(x_{1}^{k}+s x_{2}^{k}, x_{1}^{n-k}\right)=2^{k} \sum_{j_{1}, j_{2}, \ldots, j_{k} \in\{1,2\}} f\left(x_{j_{1} 1}, x_{j_{2} 2}, \ldots, x_{j_{k} k}, x_{1}^{n-k}\right)
$$

for all $x_{1}^{k}, x_{2}^{k} \in V^{k}$ and $x_{1}^{n-k} \in V^{n-k}$. In the light of [32, Theorem 3], we see that $f$ is quadratic in each of the $k$ first variables. Furthermore, by putting $x_{2}^{k}=(0, \ldots, 0)$ in $(2.1)$ and using Lemma 2.3, we have

$$
2^{k} \sum_{t \in\{-1,1\}^{n-k}} f\left(x_{1}^{k}, 2 x_{1}^{n-k}+t x_{2}^{n-k}\right)=2^{k} \sum_{m=0}^{n-k} 2^{n-k-m} 12^{m} f\left(x_{1}^{k}, \mathcal{M}_{m}^{n-k}\right)
$$

for all $x_{1}^{k} \in V^{k}$ and $x_{1}^{n-k}, x_{2}^{n-k} \in V^{n-k}$, and thus [10, Proposition 2.3] now completes the proof.

\section{Stability of (2.1)}

In this section, we prove the generalized Hyers-Ulam stability of Eq. (2.1) by a fixed point result (Theorem 3.1) in Banach spaces. Throughout, for two sets $X$ and $Y$, the set of all mappings from $X$ to $Y$ is denoted by $Y^{X}$. Here, we introduce the oncoming three hypotheses:

(A1) $Y$ is a Banach space, $\mathcal{S}$ is a nonempty set, $j \in \mathbb{N}, g_{1}, \ldots, g_{j}: \mathcal{S} \longrightarrow \mathcal{S}$ and $L_{1}, \ldots, L_{j}: \mathcal{S} \longrightarrow \mathbb{R}_{+}$,

(A2) $\mathcal{T}: Y^{\mathcal{S}} \longrightarrow Y^{\mathcal{S}}$ is an operator satisfying the inequality

$$
\|\mathcal{T} \lambda(x)-\mathcal{T} \mu(x)\| \leq \sum_{i=1}^{j} L_{i}(x)\left\|\lambda\left(g_{i}(x)\right)-\mu\left(g_{i}(x)\right)\right\|, \quad \lambda, \mu \in Y^{\mathcal{S}}, x \in \mathcal{S}
$$

(A3) $\Lambda: \mathbb{R}_{+}^{\mathcal{S}} \longrightarrow \mathbb{R}_{+}^{\mathcal{S}}$ is an operator defined through

$$
\Lambda \delta(x):=\sum_{i=1}^{j} L_{i}(x) \delta\left(g_{i}(x)\right) \quad \delta \in \mathbb{R}_{+}^{\mathcal{S}}, x \in \mathcal{S}
$$

To achieve our aim in this section, we present the next theorem which is a fundamental result in fixed point theory [13, Theorem 1].

Theorem 3.1 Let hypotheses (A1)-(A3) hold and the function $\theta: \mathcal{S} \longrightarrow \mathbb{R}_{+}$and the mapping $\phi: \mathcal{S} \longrightarrow Y$ fulfill the following two conditions:

$$
\|\mathcal{T} \phi(x)-\phi(x)\| \leq \theta(x), \quad \theta^{*}(x):=\sum_{l=0}^{\infty} \Lambda^{l} \theta(x)<\infty \quad(x \in \mathcal{S}) .
$$

Then there exists a unique fixed point $\psi$ of $\mathcal{T}$ such that

$$
\|\phi(x)-\psi(x)\| \leq \theta^{*}(x) \quad(x \in \mathcal{S}) .
$$

Moreover, $\psi(x)=\lim _{l \rightarrow \infty} \mathcal{T}^{l} \phi(x)$ for all $x \in \mathcal{S}$. 
Here and subsequently, for the mapping $f: V^{n} \longrightarrow W$, we consider the difference operator $\mathcal{D}_{Q}^{C} f: V^{n} \times V^{n} \longrightarrow W$ by

$$
\begin{aligned}
\mathcal{D}_{Q}^{C} f\left(x_{1}, x_{2}\right):= & \sum_{s \in\{-1,1\}^{k}} \sum_{t \in\{-1,1\}^{n-k}} f\left(x_{1}^{k}+s x_{2}^{k}, 2 x_{1}^{n-k}+t x_{2}^{n-k}\right) \\
& -2^{k} \sum_{m=0}^{n-k} 2^{n-k-m} 12^{m} \sum_{i \in\{1,2\}} f\left(x_{i}^{k}, \mathcal{M}_{m}^{n-k}\right)
\end{aligned}
$$

for all $x_{i}^{k}=\left(x_{i 1}, \ldots, x_{i k}\right) \in V^{k}$ and $x_{i}^{n-k}=\left(x_{i, k+1}, \ldots, x_{i n}\right) \in V^{n-k}$, where $i \in\{1,2\}$.

We recall the upcoming lemma from [3] will be useful in the proof of our stability result. For simplicity, given an $m \in \mathbb{N}$, we write $S:=\{0,1\}^{m}$, and $S_{i}$ stands for the set of all elements of $S$ having exactly $i$ zeros, i.e.,

$$
S_{i}:=\left\{\left(s_{1}, \ldots, s_{m}\right) \in S: \operatorname{card}\left\{j: s_{j}=0\right\}=i\right\}, \quad i \in\{0, \ldots, m\} .
$$

Lemma 3.2 Let $m \in \mathbb{N}, l \in \mathbb{N}_{0}$ and $\psi: S \longrightarrow \mathbb{R}$. Then

$$
\sum_{v=0}^{m} \sum_{w=0}^{m} \sum_{s \in S_{w}} \sum_{t \in S_{v}}\left(2^{l}-1\right)^{w} \psi(s t)=\sum_{i=0}^{m} \sum_{p \in S_{i}}\left(2^{l+1}-1\right)^{i} \psi(p) .
$$

From now on, $S$ stands for $\{0,1\}^{k}$ and $S_{i} \subseteq S$ for $i \in\{0, \ldots, k\}$. We have the following stability result for the functional equation (2.1).

Theorem 3.3 Let $V$ be a linear space and $W$ be a Banach space. Suppose that $\phi: V^{n} \times$ $V^{n} \longrightarrow \mathbb{R}_{+}$is a mapping satisfying the equality

$$
\lim _{l \rightarrow \infty}\left(\frac{1}{2^{3 n-k}}\right)^{l} \sum_{i=0}^{k} \sum_{p \in S_{i}}\left(2^{l}-1\right)^{i} \phi\left(2^{l}\left(p x_{1}^{k}, x_{1}^{n-k}\right), 2^{l}\left(p x_{2}^{k}, x_{2}^{n-k}\right)\right)=0
$$

for all $x_{1}, x_{2} \in V^{n}$ and

$$
\Phi(x)=: \frac{1}{2^{4 n-2 k}} \sum_{l=0}^{n}\left(\frac{1}{2^{3 n-k}}\right)^{l} \sum_{i=0}^{k} \sum_{p \in S_{i}}\left(2^{l}-1\right)^{i} \phi\left(2^{l}\left(p x^{k}, x^{n-k}\right),\left(2^{l} p x^{k}, 0\right)\right)<\infty
$$

for all $x=\left(x^{k}, x^{n-k}\right) \in V^{n}$. Assume also $f: V^{n} \longrightarrow W$ is a mapping satisfying the inequality

$$
\left\|\mathcal{D}_{Q}^{C} f\left(x_{1}, x_{2}\right)\right\| \leq \phi\left(x_{1}, x_{2}\right)
$$

for all $x_{1}, x_{2} \in V^{n}$. Then there exists a solution $\mathcal{F}: V^{n} \longrightarrow W$ of (2.1) such that

$$
\|f(x)-\mathcal{F}(x)\| \leq \Phi(x)
$$

for all $x=\left(x^{k}, x^{n-k}\right) \in V^{n}$. If $\mathcal{F}$ satisfies the 3-power condition in the last $n-k$ variables, then it is a unique multi-quadratic-cubic mapping. 
Proof Putting $x_{1}^{k}=x_{2}^{k}=x^{k}$ and $x_{1}^{n-k}=x^{n-k}, x_{2}^{n-k}=0$ in (3.3), we have

$$
\begin{aligned}
& \left\|2^{n-k} \sum_{s \in S} f\left(2 s x^{k}, 2 x^{n-k}\right)-2^{k} \sum_{m=0}^{n-k}\left(\begin{array}{c}
n-k \\
m
\end{array}\right) 2^{n-k-m} 12^{m} 2^{k} 2^{n-k-m} f(x)\right\| \\
& \quad \leq \phi\left(x,\left(x^{k}, 0\right)\right)
\end{aligned}
$$

where $x=\left(x^{k}, x^{n-k}\right) \in V^{n}$. A computation shows that (3.5) can be rewritten as follows:

$$
\left\|2^{n-k} \sum_{s \in S} f\left(2 s x^{k}, 2 x^{n-k}\right)-2^{4 n-2 k} f(x)\right\| \leq \phi\left(x,\left(x^{k}, 0\right)\right) .
$$

The above inequality implies that

$$
\left\|f(x)-\frac{1}{2^{3 n-k}} \sum_{s \in S} f\left(2 s x^{k}, 2 x^{n-k}\right)\right\| \leq \frac{1}{2^{4 n-2 k}} \phi\left(x,\left(x^{k}, 0\right)\right)
$$

for all $x=\left(x^{k}, x^{n-k}\right) \in V^{n}$. Set $\xi(x):=\frac{1}{2^{4 n-2 k}} \phi\left(x,\left(x^{k}, 0\right)\right)$ and $\mathcal{T} \xi(x):=\frac{1}{2^{3 n-k}} \sum_{s \in S} \xi\left(2 s x^{k}\right.$, $\left.2 x^{n-k}\right)$ where $\xi \in W^{V^{n}}$ and $x \in V^{n}$. Then Eq. (3.6) can be modified as

$$
\|f(x)-\mathcal{T} f(x)\| \leq \xi(x) \quad\left(x \in V^{n}\right)
$$

Define $\Lambda \eta(x):=\frac{1}{2^{3 n-k}} \sum_{s \in S} \eta\left(2 s x^{k}, 2 x^{n-k}\right)$ for all $\eta \in \mathbb{R}_{+}^{V^{n}}, x=\left(x^{k}, x^{n-k}\right) \in V^{n}$. We now see that $\Lambda$ has the form described in (A3). Furthermore, for each $\lambda, \mu \in W^{V^{n}}$ and $x \in V^{n}$, we get

$$
\begin{aligned}
\|\mathcal{T} \lambda(x)-\mathcal{T} \mu(x)\| & =\left\|\frac{1}{2^{3 n-k}}\left[\sum_{s \in S}\left(\lambda\left(2 s x^{k}, 2 x^{n-k}\right)-\mu\left(2 s x^{k}, 2 x^{n-k}\right)\right)\right]\right\| \\
& \leq \frac{1}{2^{3 n-k}} \sum_{s \in S}\left\|\lambda\left(2 s x^{k}, 2 x^{n-k}\right)-\mu\left(2 s x^{k}, 2 x^{n-k}\right)\right\| .
\end{aligned}
$$

The above relation shows that the hypothesis (A2) holds. By induction on $l$, one can check for any $l \in \mathbb{N}_{0}$ and $x \in V^{n}$ that

$$
\Lambda^{l} \xi(x):=\left(\frac{1}{2^{3 n-k}}\right)^{l} \sum_{i=0}^{k}\left(2^{l}-1\right)^{i} \sum_{p \in S_{i}} \xi\left(2^{l}\left(p x^{k}, x^{n-k}\right)\right) .
$$

Fix an $x \in V^{n}$. Here, we adopt the convention that $0^{0}=1$. Hence, (3.8) is trivially true for $l=0$. Next, assume that (3.8) holds for a $l \in \mathbb{N}_{0}$. Then, by using Lemma 3.2 for $m=n$ and $\psi(s):=\xi\left(2^{l+1}\left(s x^{k}, x^{n-k}\right)\right)(s \in S)$, we get

$$
\begin{aligned}
\Lambda^{l+1} \xi(x) & =\Lambda\left(\Lambda^{l} \xi\right)(x) \\
& =\frac{1}{2^{3 n-k}} \sum_{\nu=0}^{k} \sum_{t \in S_{v}}\left(\Lambda^{l} \xi\right)\left(2 t x^{k}, 2 x^{n-k}\right) \\
& =\left(\frac{1}{2^{3 n-k}}\right)^{l+1} \sum_{v=0}^{k} \sum_{t \in S_{v}} \sum_{w=0}^{k}\left(2^{l}-1\right)^{w} \sum_{s \in S_{w}} \xi\left(2^{l+1}\left(s t x^{k}, x^{n-k}\right)\right)
\end{aligned}
$$




$$
\begin{aligned}
& =\left(\frac{1}{2^{3 n-k}}\right)^{l+1} \sum_{v=0}^{k} \sum_{w=0}^{k} \sum_{s \in S_{w}} \sum_{t \in S_{v}}\left(2^{l}-1\right)^{w} \xi\left(2^{l+1}\left(s t x^{k}, x^{n-k}\right)\right) \\
& =\left(\frac{1}{2^{3 n-k}}\right)^{l+1} \sum_{i=0}^{k} \sum_{p \in S_{i}}\left(2^{l+1}-1\right)^{i} \xi\left(2^{l+1}\left(p x^{k}, x^{n-k}\right)\right) .
\end{aligned}
$$

Therefore, (3.8) holds for any $l \in \mathbb{N}_{0}$ and $x \in V^{n}$. Now, (3.2) and (3.8) necessitate that all assumptions of Theorem 3.1 are satisfied. Hence, there exists a mapping $\mathcal{F}: V^{n} \longrightarrow W$ such that

$$
\mathcal{F}(x)=\lim _{l \rightarrow \infty}\left(\mathcal{T}^{l} f\right)(x)=\frac{1}{2^{3 n-k}} \sum_{s \in S} \mathcal{F}\left(2\left(s x^{k}, x^{n-k}\right)\right) \quad\left(x \in V^{n}\right),
$$

and also (3.4) holds. We shall to show that

$$
\left\|\mathcal{D}_{Q}^{C}\left(\mathcal{T}^{l} f\right)\left(x_{1}, x_{2}\right)\right\| \leq\left(\frac{1}{2^{3 n-k}}\right)^{l} \sum_{i=0}^{k} \sum_{p \in S_{i}}\left(2^{l}-1\right)^{i} \phi\left(2^{l}\left(p x_{1}^{k}, x_{1}^{n-k}\right), 2^{l}\left(p x_{2}^{k}, x_{2}^{n-k}\right)\right)
$$

for all $x_{1}=\left(x_{1}^{k}, x_{1}^{n-k}\right), x_{2}=\left(x_{2}^{k}, x_{2}^{n-k}\right) \in V^{n}$ and $l \in \mathbb{N}_{0}$. We argue by induction on $l$. It is easy to see that inequality (3.9) is valid for $l=0$ by (3.3). Assume that (3.9) is true for an $l \in \mathbb{N}_{0}$. For each $x_{1}=\left(x_{1}^{k}, x_{1}^{n-k}\right), x_{2}=\left(x_{2}^{k}, x_{2}^{n-k}\right) \in V^{n}$, we have

$$
\begin{aligned}
& \left\|\mathcal{D}_{Q}^{C}\left(\mathcal{T}^{l+1} f\right)\left(x_{1}, x_{2}\right)\right\| \\
& \quad=\frac{1}{2^{3 n-k}}\left\|\sum_{s \in S} \mathcal{D}_{Q}^{C}\left(\mathcal{T}^{l} f\right)\left(2 s x_{1}^{k}, 2 x_{1}^{n-k}, 2 s x_{2}^{k}, 2 x_{2}^{n-k}\right)\right\| \\
& \quad \leq\left(\frac{1}{2^{3 n-k}}\right)^{l+1} \sum_{s \in S} \sum_{i=0}^{k} \sum_{t \in S_{i}}\left(2^{l}-1\right)^{i} \phi\left(2^{l+1}\left(s t x_{1}^{k}, x_{1}^{n-k}\right), 2^{l+1}\left(s t x_{2}^{k}, x_{2}^{n-k}\right)\right) \\
& \quad=\left(\frac{1}{2^{3 n-k}}\right)^{l+1} \sum_{i=0}^{k} \sum_{p \in S_{i}}\left(2^{l+1}-1\right)^{i} \phi\left(2^{l+1}\left(p x_{1}^{k}, x_{1}^{n-k}\right), 2^{l+1}\left(p x_{2}^{k}, x_{2}^{n-k}\right)\right)
\end{aligned}
$$

for all $x_{1}, x_{2} \in V^{n}$. We note that the last equality follows from Lemma 3.2 with $m:=k$ and $\psi(s):=\phi\left(2^{l+1}\left(s x_{1}^{k}, x_{1}^{n-k}\right), 2^{l+1}\left(s x_{2}^{k}, x_{2}^{n-k}\right)\right)(s \in S)$. Letting $l \rightarrow \infty$ in (3.9) and applying (3.1), we arrive at $\mathcal{D}_{Q}^{C} \mathcal{F}\left(x_{1}, x_{2}\right)=0$ for all $x_{1}, x_{2} \in V^{n}$. This means that the mapping $\mathcal{F}$ satisfies (2.1). Finally, assume that $\mathfrak{F}: V^{n} \longrightarrow W$ is another mapping satisfying Eq. (2.1) and inequality (3.4), and fix $x \in V^{n}, j \in \mathbb{N}$. Then, by Lemma 2.3 and (3.2), we have

$$
\begin{aligned}
& \|\mathcal{F}(x)-\mathfrak{F}(x)\| \\
& \quad=\left\|\left(\frac{1}{2^{3 n-k}}\right)^{j} \mathcal{F}\left(2^{j} x\right)-\left(\frac{1}{2^{3 n-k}}\right)^{j} \mathfrak{F}\left(2^{j} x\right)\right\| \\
& \quad \leq\left(\frac{1}{2^{3 n-k}}\right)^{j}\left(\left\|\mathcal{F}\left(2^{j} x\right)-f\left(2^{j} x\right)\right\|+\left\|\mathfrak{F}\left(2^{j} x\right)-f\left(2^{j} x\right)\right\|\right)
\end{aligned}
$$




$$
\begin{aligned}
& \leq \frac{2}{2^{4 n-2 k}}\left(\frac{1}{2^{3 n-k}}\right)^{j} \Phi\left(2^{j} x\right) \\
& \leq \frac{1}{2^{4 n-2 k-1}}\left(\frac{1}{2^{3 n-k}}\right)^{j} \sum_{l=j}^{\infty}\left(\frac{1}{2^{3 n-k}}\right)^{l} \sum_{i=0}^{k} \sum_{p \in S_{i}}\left(2^{l}-1\right)^{i} \phi\left(2^{l}\left(p x^{k}, x^{n-k}\right),\left(2^{l} p x^{k}, 0\right)\right) .
\end{aligned}
$$

Consequently, letting $j \rightarrow \infty$ and using the fact that the series (3.2) is convergent for all $x \in V^{n}$, we obtain $\mathcal{F}(x)=\mathfrak{F}(x)$ for all $x \in V^{n}$, and thus the proof is now finished.

In the next corollary, we show that the functional equation (2.1) is stable when the norm of $\mathcal{D}_{Q}^{C} f\left(x_{1}, x_{2}\right)$ is controlled by a small positive real number $\delta$, for all $x_{1}, x_{2} \in V^{n}$.

Corollary 3.4 Let $\delta>0$. Let also $V$ be a vector space and $W$ be a Banach space. If $f$ : $V^{n} \longrightarrow W$ is a mapping satisfying the inequality

$$
\left\|\mathcal{D}_{Q}^{C} f\left(x_{1}, x_{2}\right)\right\| \leq \delta
$$

for all $x_{1}, x_{2} \in V^{n}$, then there exists a solution $\mathcal{F}: V^{n} \longrightarrow W$ of (2.1) such that

$$
\|f(x)-\mathcal{F}(x)\| \leq \frac{\delta}{2^{n-k}\left(2^{3 n-k}-1\right)}
$$

for all $x \in V^{n}$. Moreover, if $\mathcal{F}$ satisfies the 3-power condition in the last $n-k$ variables, then it is a unique multi-quadratic-cubic mapping.

Proof Setting the constant function $\phi\left(x_{1}, x_{2}\right)=\delta$ for all $x_{1}, x_{2} \in V^{n}$, and applying Theorem 3.3, we have

$$
\begin{aligned}
\Phi(x) & =\frac{1}{2^{4 n-2 k}} \sum_{l=0}^{\infty}\left(\frac{1}{2^{3 n-k}}\right)^{l} \sum_{i=0}^{k} \sum_{p \in S_{i}}\left(2^{l}-1\right)^{i} \phi\left(2^{l}\left(p x^{k}, x^{n-k}\right),\left(2^{l} p x^{k}, 0\right)\right) \\
& =\frac{\delta}{2^{4 n-2 k}} \sum_{l=0}^{\infty}\left(\frac{1}{2^{3 n-k}}\right)^{l} \sum_{i=0}^{k}\left(\begin{array}{l}
k \\
i
\end{array}\right)\left(2^{l}-1\right)^{i} \times 1^{k-i} \\
& =\frac{\delta}{2^{4 n-2 k}} \sum_{l=0}^{\infty}\left(\frac{1}{2^{3 n-k}}\right)^{l} 2^{k l} \\
& =\frac{\delta}{2^{4 n-2 k}} \sum_{l=0}^{\infty}\left(\frac{1}{2^{3 n-k}}\right)^{l} \\
& =\frac{\delta}{2^{n-k}\left(2^{3 n-k}-1\right)}
\end{aligned}
$$

Under some conditions the functional equation (2.1) can be hyperstable as follows.

Corollary 3.5 Suppose that $\delta_{i j}>0$ for $i \in\{1,2\}$ and $j \in\{1, \ldots, n\}$ fulfill $\sum_{i=1}^{2} \sum_{j=1}^{n} \delta_{i j}<$ $3 n-k$. Let $V$ be a normed space and $W$ be a Banach space. If $: V^{n} \rightarrow W$ is a mapping satisfying the inequality

$$
\left\|\mathcal{D}_{Q}^{C} f\left(x_{1}, x_{2}\right)\right\| \leq \prod_{i=1}^{2} \prod_{j=1}^{n}\left\|x_{i j}\right\|^{\delta_{i j}}
$$


for all $x_{1}, x_{2} \in V^{n}$, then $f$ satisfies Eq. (2.1). In particular, iff satisfies the 3-power condition in the last $n-k$ variables, then it is a multi-quadratic-cubic mapping.

Proof The result follows immediately from Proposition 2.4 and Theorem 3.3.

Putting $k=n$ in Theorem 3.3, we obtain [3, Corollary 2] on the stability of multiquadratic mappings. Furthermore, by considering $k=0$ in Theorem 3.3, we obtain the below result on the stability of multi-cubic mappings.

Corollary 3.6 Let $\delta>0$. Let also $V$ be a vector space and $W$ be a Banach space. Suppose that $f: V^{n} \longrightarrow W$ is a mapping satisfying the inequality

$$
\left\|\sum_{t \in\{-1,1\}^{n}} f\left(2 x_{1}+t x_{2}\right)-\sum_{k=0}^{n} 2^{n-k} 12^{k} f\left(\mathcal{M}_{k}^{n}\right)\right\| \leq \delta
$$

for all $x_{1}, x_{2} \in V^{n}$. Then there exists a solution $\mathcal{C}: V^{n} \longrightarrow W$ of (2.1) when $k=0$ such that

$$
\|f(x)-\mathcal{C}(x)\| \leq \frac{\delta}{2^{n}\left(2^{3 n}-1\right)}
$$

for all $x \in V^{n}$. In addition, if $\mathcal{C}$ satisfies the 3-power condition in each variable, then it is a unique multi-cubic mapping.

\section{Acknowledgements}

The authors sincerely thank the anonymous reviewer for her/his careful reading, constructive comments and suggesting some related references to improve the quality of the first draft of paper.

\section{Funding}

Not applicable.

\section{Availability of data and materials}

Not applicable. In fact, all results are obtained without any software and found by manual computations. In other words, the manuscript is in the pure mathematics (mathematical analysis) category.

\section{Competing interests}

The authors declare that they have no competing interests.

\section{Authors' contributions}

The authors conceived of the study, participated in its design and coordination, drafted the manuscript, participated in the sequence alignment, and read and approved the final manuscript.

\section{Author details}

'Department of Mathematics, Garmsar Branch, Islamic Azad University, Garmsar, Iran. ${ }^{2}$ Faculty of Management,

University of Primorska, Cankarjeva 5, SI-6000 Koper, Slovenia.

\section{Publisher's Note}

Springer Nature remains neutral with regard to jurisdictional claims in published maps and institutional affiliations.

Received: 1 December 2020 Accepted: 26 February 2021 Published online: 10 March 2021

\section{References}

1. Aoki, T.: On the stability of the linear transformation in Banach spaces. J. Math. Soc. Jpn. 2, 64-66 (1950)

2. Bahyrycz, A., Ciepliński, K., Olko, J.: On an equation characterizing multi Cauchy-Jensen mappings and its Hyers-Ulam stability. Acta Math. Sci. Ser. B Engl. Ed. 35, 1349-1358 (2015)

3. Bahyrycz, A., Ciepliński, K., Olko, J.: On an equation characterizing multi-additive-quadratic mappings and its Hyers-Ulam stability. Appl. Math. Comput. 265, 448-455 (2015)

4. Bodaghi, A.: Cubic derivations on Banach algebras. Acta Math. Vietnam. 38(4), 517-528 (2013)

5. Bodaghi, A.: Intuitionistic fuzzy stability of the generalized forms of cubic and quartic functional equations. J. Intell. Fuzzy Syst. 30, 2309-2317 (2016) 
6. Bodaghi, A., Alias, I.A., Ghahramani, M.H.: Approximately cubic functional equations and cubic multipliers. J. Inequal. Appl. 2011, 53 (2011)

7. Bodaghi, A., Moosavi, S.M., Rahimi, H.: The generalized cubic functional equation and the stability of cubic Jordan *-derivations. Ann. Univ. Ferrara 59, 235-250 (2013)

8. Bodaghi, A., Park, C., Mewomo, O.T:: Multiquartic functional equations. Adv. Differ. Equ. 2019, 312 (2019). https://doi.org/10.1186/s13662-019-2255-5

9. Bodaghi, A., Park, C., Yun, S.: Almost multi-quadratic mappings in non-Archimedean spaces. AlMS Math. 5(5), 5230-5239 (2020). https://doi.org/10.3934/math.2020336

10. Bodaghi, A., Shojaee, B.: On an equation characterizing multi-cubic mappings and its stability and hyperstability. Fixed Point Theory. To appear. arXiv:1907.09378v2

11. Bourgin, D.G.: Approximately isometric and multiplicative transformations on continuous function rings. Duke Math J. 16, 385-397 (1949)

12. Brzdesk, J.: Stability of the equation of the p-Wright affine functions. Aequ. Math. 85, 497-503 (2013)

13. Brzdęk, J., Chudziak, J., Páles, Z.: A fixed point approach to the stability of functional equations in non-Archimedean metric spaces. Nonlinear Anal. 74, 6728-6732 (2011)

14. Ciepliński, K.: Generalized stability of multi-additive mappings. Appl. Math. Lett. 23, 1291-1294 (2010)

15. Ciepliński, K.: On the generalized Hyers-Ulam stability of multi-quadratic mappings. Comput. Math. Appl. 62 , 3418-3426 (2011)

16. Ciepliński, K:: On Ulam stability of a functional equation. Results Math. 75, 151 (2020)

17. Dashti, M., Khodaei, H.: Stability of generalized multi-quadratic mappings in Lipschitz spaces. Results Math. 74, 163 (2019)

18. Dashti, M., Khodaei, H.: A new type of approximation for cubic functional equations in Lipschitz spaces. Int. J. Nonlinear Anal. Appl. 11, 291-300 (2020)

19. Ebrahimi Hoseinzadeh, N., Bodaghi, A., Mardanbeigi, M.R.: Almost multi-cubic mappings and a fixed point application. Sahand Commun. Math. Anal. 17(3), 131-143 (2020)

20. Găvruța, P.: A generalization of the Hyers-Ulam-Rassias stability of approximately additive mappings. J. Math. Anal. Appl. 184, 431-436 (1994)

21. Hyers, D.H.: On the stability of the linear functional equation. Proc. Natl. Acad. Sci. USA 27, 222-224 (1941)

22. Jun, K.W., Kim, H.M.: The generalized Hyers-Ulam-Rassias stability of a cubic functional equation. J. Math. Anal. Appl. $274(2), 267-278(2002)$

23. Jun, K.W., Kim, H.M.: On the Hyers-Ulam-Rassias stability of a general cubic functional equation. Math. Inequal. Appl. 6(2), 289-302 (2003)

24. Kuczma, M.: An Introduction to the Theory of Functional Equations and Inequalities. Cauchy's Equation and Jensen's Inequality. Birkhäuser, Basel (2009)

25. Maksa, G., Páles, Z.: Hyperstability of a class of linear functional equations. Acta Math. 17(2), 107-112 (2001)

26. Park, C., Bodaghi, A.: Two multi-cubic functional equations and some results on the stability in modular spaces. J. Inequal. Appl. 2020, 6 (2020). https://doi.org/10.1186/s13660-019-2274-5

27. Rassias, J.M.: On approximately of approximately linear mappings by linear mappings. J. Funct. Anal. USA 46, 126-130 (1982)

28. Rassias, J.M.: Solution of the Ulam stability problem for cubic mappings. Glas. Mat. Ser. III 36(1), 63-72 (2001)

29. Rassias, T.M.: On the stability of the linear mapping in Banach space. Proc. Am. Math. Soc. 72(2), 297-300 (1978)

30. Salimi, S., Bodaghi, A.: A fixed point application for the stability and hyperstability of multi-Jensen-quadratic mappings. J. Point Theory Appl. 22, 9 (2020). https://doi.org/10.1007/s11784-019-0738-3

31. Ulam, S.M.: Problems in Modern Mathematics. Science Editions, Wiley, New York (1964)

32. Zhao, X., Yang, X., Pang, C.-T.: Solution and stability of the multiquadratic functional equation. Abstr. Appl. Anal. 2013, Article ID 415053 (2013)

\section{Submit your manuscript to a SpringerOpen ${ }^{\circ}$ journal and benefit from:}

- Convenient online submission

- Rigorous peer review

- Open access: articles freely available online

- High visibility within the field

- Retaining the copyright to your article

Submit your next manuscript at $\gg$ springeropen.com 